\title{
SAAPIENS: Uma ferramenta de Autoria de Objetos de Aprendizagem e Apoio Pedagógico na Dedução Natural na Lógica Proposicional
}

\author{
Agnaldo Martins Rodrigues ${ }^{1}$, Matheus Campezatto Galão ${ }^{1}$, Gustavo Schwarz ${ }^{1}$, \\ João Carlos Gluz ${ }^{1}$
}

${ }^{1}$ Programa Interdisciplinar de Pós Graduação em Computação Aplicada (PIPCA) Universidade do Vale do Rio dos Sinos (UNISINOS) - Caixa Postal 275 - 93.022-000 São Leopoldo -RS

\{agnaldoesapucaia.ifsul.edu.br, matheus.cg@gmail.com, gustavo.sne@gmail. com, jcgluz@unisinos.br\}

\begin{abstract}
The need of tools to help teachers, which are able to assist them in planning, implementation and monitoring of the teaching-learning process, is an important necessity in the educational area. In some cases, difficulties to access to these technologies, coupled with the lack of knowledge and understanding on the part of teachers, undermines the way in which educational contents could be developed and used. This is the main motivation behind the creation of SAAPIEnS authoring and monitoring. The tool has a multiagent system architecture built over the Heraclito tutoring system. It makes possible for teachers to create customizable learning objects for the learning domain of natural deduction in propositional logic. It also helps teachers to check and monitor how much the authored learning objects are contributing to the improvement and progress of learners.
\end{abstract}

Resumo. A necessidade de ferramentas que auxiliem os professores, no planejamento, implementação e no monitoramento do processo de ensinoaprendizagem, é uma realidade importante na área educacional. Em alguns casos, as dificuldades de acesso a essas tecnologias, juntamente com a falta de conhecimento e compreensão por parte dos professores, prejudica a forma como conteúdos educacionais poderiam ser desenvolvidos e utilizados. Esta é a principal motivação por trás da criação do SAAPIEnS ferramenta de autoria e acompanhamento educacional. A ferramenta tem uma arquitetura de sistema multiagente construída sobre o sistema Tutor Heráclito. Isso possibilita aos professores criar objetos de aprendizagem personalizáveis para o domínio de aprendizagem na dedução natural na lógica proposicional. Ele também ajuda os professores a verificar e monitorar o quanto os objetos de aprendizagem estão contribuindo para a melhoria e progresso dos alunos.

\section{Introdução}

O uso de ferramentas de autoria e acompanhamento de conteúdos digitais, em particular para materiais em formato de Objetos de Aprendizagem (OA), que sejam adequadamente projetados à professores em ambientes digitais de ensino (sejam ambientes Web ou não), potencialmente exige um maior conhecimento técnico na criação destes conteúdos. 
Esta exigência, evidentemente, pode gerar barreiras no uso desse tipo de material digital por parte dos professores. Uma pressuposição primordial do presente trabalho é que professores podem e devem se tornar autores de conteúdos digitais instrucionais, mas sem a necessidade de habilidades de programação ou treinamento técnico extensivo na área de Tecnologias de Informação e Comunicação (TIC). Também se espera que os professores possam ter acesso às informações sobre o uso destes objetos, tais como registros nos bancos de dados dos ambientes de ensino, sem necessitar de conhecimentos técnicos aprofundados.

Neste contexto, o presente trabalho buscou centrar sua pesquisa sobre ferramentas de apoio a autoria e acompanhamento de conteúdos digitais baseadas em infraestruturas previamente existentes. A infraestrutura escolhida foi o sistema de tutoria Heráclito [Penteado e Gluz, 2011], que é voltada ao ensino de Lógica Proposicional através da combinação das tecnologias de agentes inteligentes e OA. Este sistema vem sendo aplicado em processos de ensino real em sala de aula, obtendo resultados significativos [Penteado, 2012].

O objetivo final do presente trabalho é desenvolver uma de ferramenta de apoio à autoria e ao acompanhamento do uso dos Objetos de Aprendizagem Heráclito (OAH) [Mossman et al., 2012] complementando o cenário de suporte ao professor. Um OA Heráclito é formado por um arquivo em formato $J A R^{l}$ que pode ser obtido diretamente do $\mathrm{AVA}^{2}$ sendo utilizado na disciplina ou através do site do Portal OBAA-MILOS ${ }^{3}$. Este arquivo $J A R$ contém além do aplicativo Editor de Provas e de seu manual, as atividades customizadas e preparadas pelo professor com o SAAPIENS, exemplos de demonstrações corretas e materiais explicativos sobre as atividades e os conteúdos de Lógica tratados pelo objeto.

Assim sendo, esse artigo apresenta a arquitetura e a proposta da criação do sistema SAAPIEnS (Sistema de Autoria e AcomPanhamento Inteligente de Ensino Superior - módulo compatível com OA Heráclito) que tem como objetivo fornecer suporte ao professor através de uma ferramenta de autoria que facilite a criação, manutenção e reutilização dos $\mathrm{OAH}$ e que possibilite também, o acompanhamento contínuo do uso destes objetos pelos alunos através de um conjunto de análises e estatísticas disponibilizadas pela ferramenta.

Esse artigo está organizado da seguinte maneira: na seção 2 serão apresentados os conceitos básicos e arquitetura da ferramenta, seção 3 detalha os subsistemas que compõe a ferramenta. Na seção 4 é apresentada a aplicação e contribuição da ferramenta, na seção 5 as considerações finais.

\section{Sistemas de Autoria}

O conceito de ferramentas de criação de conteúdos é um termo genérico que se refere a um software para produção de conteúdo digital. A W3C(World Wide Web Consortium) (W3C, 2014) define ferramenta de autoria como, qualquer software, ou coleção de componentes de software, que os autores podem usar para criar ou modificar o conteúdo e disponibilizá-lo para uso por outras pessoas. O termo "Authoring Tools" é comumente referenciado como um software para autores de conteúdo ou sistemas de

\footnotetext{
${ }^{1}$ JAR Java ARchieve

2 AVA Ambiente Virtual de Aprendizagem

${ }^{3}$ O Portal OBAA-MILOS está disponível em: http://obaa.unisinos.br/.
} 
autoria. Esta dualidade terminológica refere-se a duas abordagens: a primeira que destaca o lado tecnológico, que estão diretamente relacionadas às principais funcionalidades das ferramentas de autoria e a outra aos aspectos pedagógicos, como fatores importantes na fase de concepção do OA. Na Figura 1 são apresentadas as características que envolvem cada um dos aspectos mencionados.

\section{Sistema de Autoria}

\section{Aspectos Tecnológicos}

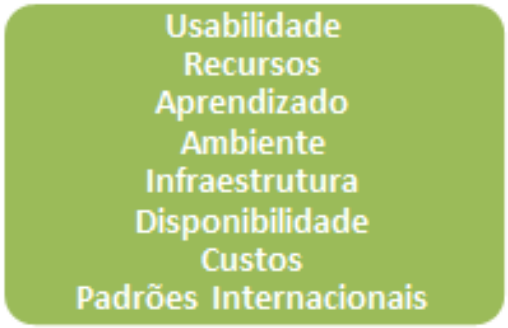

\section{Aspectos Pedagógicos}

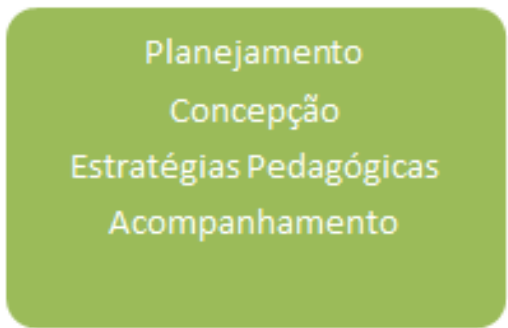

Figura 1. Abordagens envolvidas em um Sistema de Autoria Fonte: Autor

Embora exista uma diversidade tecnológica de recursos à disposição, o nível de conhecimento exigido por parte do autor ainda é alto e trabalhoso. O processo de planejamento e construção de materiais didático-pedagógicos em formato digital envolve uma série de requisitos essenciais e muitas vezes, acaba inviabilizando o seu desenvolvimento por parte do autor, fazendo com que muitos projetos acabem sendo descontinuados ou abandonados. Em algumas situações, a solução é o desenvolvimento e a utilização de uma ferramenta customizada, que atenda a uma determinada demanda em um domínio específico, como é o caso do SAAPIEnS objeto desse trabalho.

\section{Acompanhamento/Monitoração de Atividades Pedagógicas}

O Acompanhamento Pedagógico é um elemento estruturante em processos de formação, que visa assegurar a qualidade do ensino pretendido no contexto referenciado. Numa perspectiva multi-escalar e inter-relacional envolvendo professores, alunos e o próprio ambiente, visa estimular a inovação pedagógica e fortalecer os processos de ensino dinamicamente, gerando novas concepções e práticas educativas. É assumido como a possibilidade de qualificar mais o processo de ensino, por meio do planejamento e das ações. Os elementos principais que são considerados nesse contexto é a organização, planejamento do material e captação dessas experiências. Algumas ferramentas foram estudadas e entre elas gostaria de citar duas pelo fato de apresentarem como características, um processo de acompanhamento e monitoramento da aprendizagem.

O SEstatNet (Nassar et al, 2014) é uma ferramenta que usa internet como recurso, num ambiente flexível de ensino-aprendizagem. Ele é voltado basicamente para o ensino da estatística, no qual é disponibilizado um conjunto de recursos que possibilita o aprendizado. Nele o estudante planeja e realiza uma pesquisa, de forma a obter os dados que servirão de objeto de estudo. O sistema trabalha com metodologias ativas de aprendizagem, onde em cada sessão, o estudante possa aprender os principais conceitos estatísticos aplicando-os aos seus próprios dados. 
O processo de aprendizagem é livre, no qual os conteúdos não possuem uma seqüência pré-estabelecida de apresentação. $O$ suporte pode ser realizado através de um o mapa conceitual, onde o aluno encontra explicitado o raciocínio estatístico da análise dos dados. O controle de acompanhamento e monitoramento é realizado por intermédio de análises dos $\log s$ gerados durante a interação do aluno com a aplicação. Existe um sistema de Auto avaliação com auxílio de um Agente Pedagógico Animado que auxilia no processo. O professor também tem acesso à um conjunto de informações, como gráficos e relatórios da trilha de aprendizado do aluno.

$\mathrm{O} M O O D L E^{4}$ é o AVA mais utilizado nas plataformas de cursos à distância na maioria das Universidades e Escolas. Entre os diversos recursos existentes, o de acompanhamento ainda é restrito às informações geradas nos relatórios de registros de interações com o ambiente. Tais como, atividades existentes nos cursos, chats, fóruns e avaliações, quizzes e outros. Porém nesses casos, é necessário dispor de Plugins desenvolvidos por terceiros para se ter uma maior e melhor qualidade na análises das informações disponibilizadas aos professores e alunos.

O SAAPIEnS possui na sua implementação, os registros das interações entre os alunos e a ferramenta, abrangendo tanto as resoluções das questões de lógica, quanto o auxílio do sistema Tutor. $\mathrm{Na}$ análise dos Logs é possível coletar informações relevantes sobre como os alunos usam a ferramenta. O que ajuda a compreender, por exemplo, o comportamento dos mesmos com relação à interface assim como melhorar a qualidade das interações na relação humano-computador. Além de contribuir de maneira significativa no processo de ensino, pois permite ao professor, definir ações pedagógicas que irão auxiliar, nas dificuldades detectadas pelos alunos, propondo assim novas atividades $(\mathrm{OAH})$, gerando assim, novas informações, num ciclo contínuo de melhoria.

\section{Arquitetura}

O Sistema SAAPIEnS é dividido em três subsistemas principais (Figura 2): Autoria, responsável pela criação e edição dos $\mathrm{OAH}$; Acompanhamento, responsável pela coleta, tratamento dos dados, controle das atividades e a geração de análises; Gerência, responsável pelo controle, parametrização e manutenção da ferramenta.

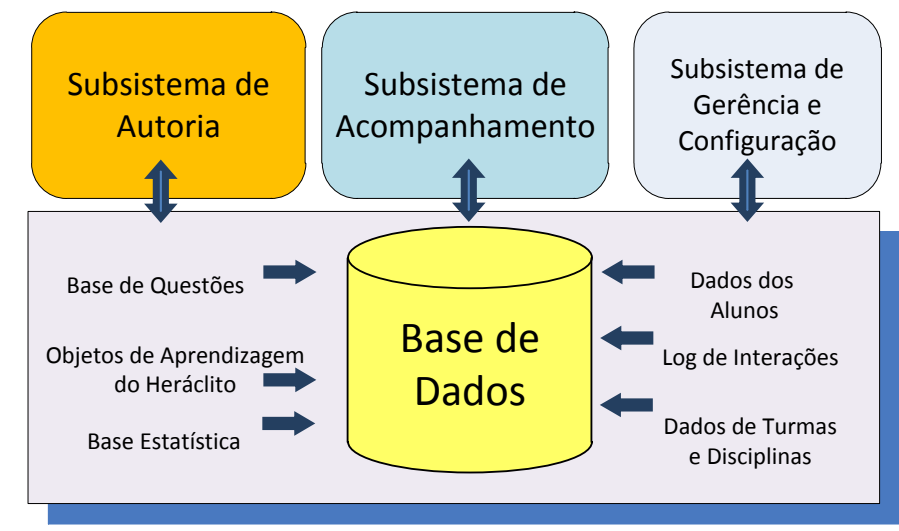

Figura 2. Arquitetura geral do Sistema SAAPIEnS. Fonte: autor

\footnotetext{
${ }^{4} \mathrm{http}: / /$ moodle.org
} 
O subsistema de Autoria implementa a ferramenta principal. É através dele que se dá o processo da concepção dos $\mathrm{OAH}$ e a disponibilização dos seus recursos. Entre algumas das facilidades que o sistema apresenta é a possibilidade de ser acessado via Web, com suporte a conteúdos educacionais multimídia, ativos e com tutoria inteligente.

O processo de criação de um $\mathrm{OAH}$ se inicia pela definição dos atributos (parâmetros) básicos do OAH (Figura 3), seguida da definição do conjunto de atividades didático-pedagógicas de acordo com os objetivos de ensino a serem alcançados pelo uso do OA, ou seja, qual o nível de conhecimento que se espera que os alunos atinjam. Essas atividades poderão ser posteriormente alteradas e customizadas.

Além dos atributos básicos de identificação do $\mathrm{OAH}$, deverão ser definidos os tipos de atividades que farão parte do objeto. Estas atividades são divididas em duas categorias distintas de materiais, o primeiro relacionado aos exercícios de lógica, destinado à prática dos alunos e o segundo, para materiais expositivos como apoio às atividades:

- Exercício de Lógica: Argumento de Dedução Natural da Lógica Proposicional (DNLP), Tabela-Verdade e Avaliação de Fórmulas;

- Material Expositivo: Textos, Imagens, Arquivos (PDF, DOC...), Material de Apresentação (arquivo ppt), Animações e outros;

Todo conjunto de atividades do OAH deve ser categorizado e classificado, podendo ser dividido em quatro níveis: 0 - Fundamental, 1-Básico, 2-Intermediário e 3Avançado de acordo com critério estabelecido pelo professor;

Uma vez definidos os parâmetros básicos e as atividades do OAH, é gerada uma identificação do objeto (ID) no SAAPIEnS. Após essa geração o professor pode customizar o perfil de ajuda para o $\mathrm{OAH}$, configurando funcionalidades de tutoria automática do Heráclito, incluindo não só a habilitação da tutoria, mas também como se dará esta tutoria de acordo com o nível de classificação das atividades do OAH. Os recursos de tutoria fornecidos ao aluno são divididos em dois níveis de suporte: o primeiro nível de suporte fornece ajuda mais genérica que pode ser a indicação um material extra para o aluno, uma explicação de provas através de exemplos ou um tutorial em vídeo. No segundo nível de suporte, o sistema fornece auxílios pontuais, como sugestão de regras de inferência a serem usadas na continuação do exercício e fornecimento de avisos quando uma regra está sendo usada de forma incorreta ou redundante. Neste caso o parâmetro Dicas indica se o Heráclito fornecerá o próximo passo da solução, enquanto que PróximoPasso que habilita Feedback passo-a-passo no processo de resolução. 


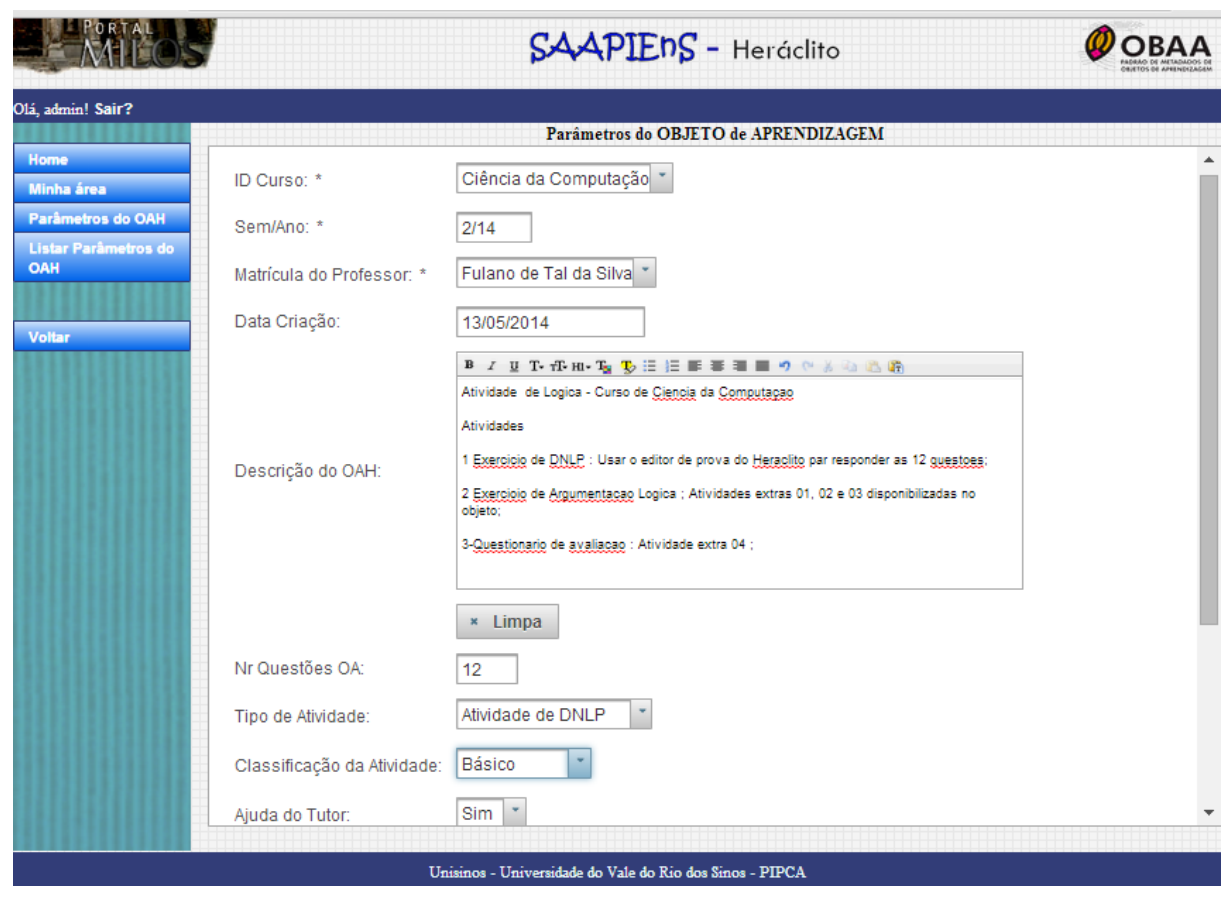

Figura 3. Tela do editor de parâmetros do OAH. Fonte: Autor

Uma vez definidos os parâmetros iniciais do objeto, o professor pode então definir o conjunto de atividades que irão compor o OAH. No subsistema de edição há uma interface de atividades extras que permite ao professor incluir outras tarefas ao $\mathrm{OAH}$, como a inserção de objetos do tipo: documentos pdf, arquivos de imagens, programas, animações ou questionários através do recurso de Upload de arquivos selecionados. O professor poderá deixar instruções aos alunos, referente à cada atividade em um espaço disponível para edição. Essas atividades poderão ser de cunho informativo ou utilizado como atividades de avaliação. Na Figura 4 é visualizada a interface de atividades extras.

Através do subsistema de Acompanhamento o professor tem acesso a um conjunto de análises e estatísticas, podendo verificar se a configuração proposta do OAH está realmente de acordo como o nível de aprendizagem desejado. Dessa forma, o professor pode fazer os ajustes necessários ao detectar falhas, dificuldades ou facilidades encontradas por parte dos alunos.

O subsistema de Acompanhamento é responsável pela coleta dos dados e das atividades feitas pelos alunos e pela geração das informações estatísticas baseadas nas análises destes dados. Para tanto, este subsistema é capaz de monitorar as interações entre os alunos e as atividades de resolução de exercícios contidos nos OAH. São os registros dessas interações que fornecerão a base para as análises e controles do subsistema de acompanhamento. 


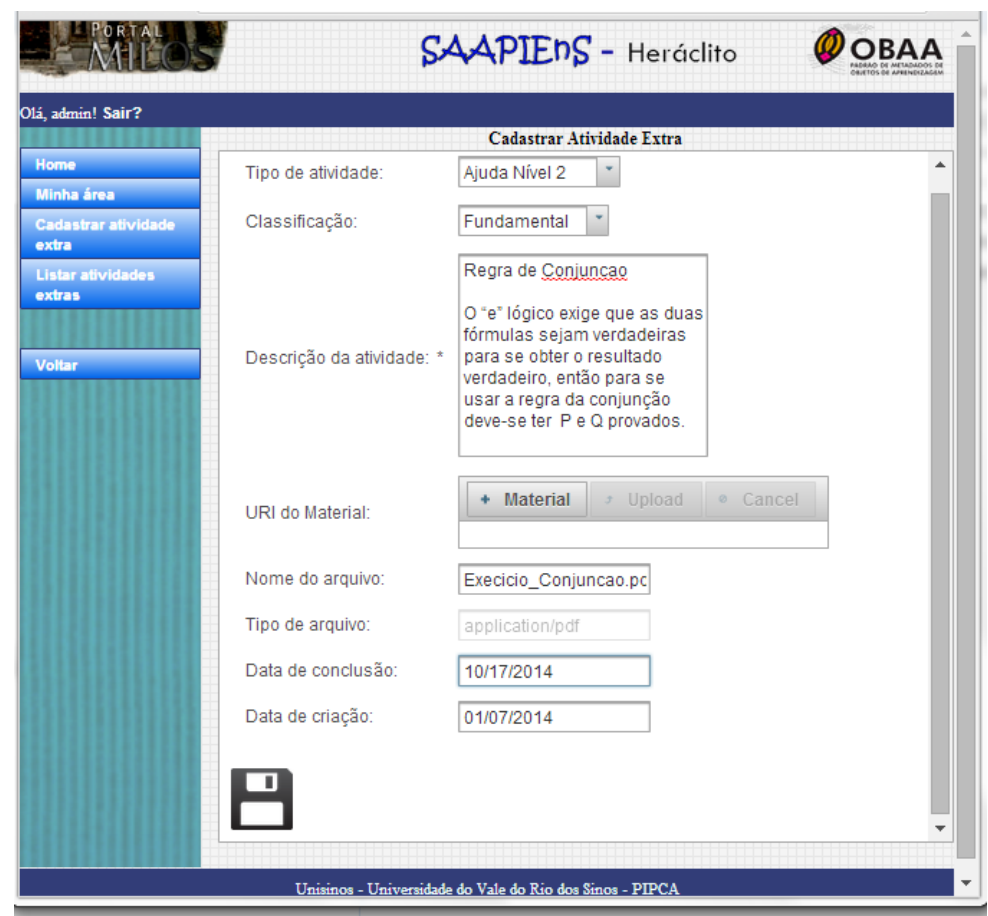

Figura 4. Inclusão de Atividades Extras Fonte: Autor

O subsistema de Gerência é o responsável pelas ações de controle e parametrização da ferramenta. Suas principais funcionalidades são: segurança dos dados, manutenção das contas e cadastros gerais, parametrização e habilitação de recursos da ferramenta, importação e exportação de arquivos e manter a consistência da base de dados.

\section{Agentes de Software}

Em termos de arquitetura o SAAPIEnS é formado por dois agentes de software [Wooldridge, 2009; Bordini et al, 2007]: o agente Monitor e o agente Analyzer. O agente Monitor tem o papel de monitorar as interações dos alunos com os OAH e atualizar as bases de dados com os registros dessas monitorações. As ações realizadas pelo aluno durante a execução das atividades são gravadas no registro $(\log )$ de atividades que servirão para análises posteriores. Entre as ações monitoradas pelo agente Monitor estão: (a) o tempo total que o aluno está conectado realizando atividade; (b) o tempo dispendido para realizar uma determinada prova seja ela parcial ou total (definese prova parcial, àquela em que o aluno não chegou ao resultado final, porém deu início a sua resolução); (c) o tempo de inatividade;(d) o número de passos que o aluno usou para realizar determinada prova; (e) uso de ajuda do tutor solicitado pelo aluno e (f) o uso de dicas fornecidas para determinada solução. O agente Monitor também controla quais as regras utilizadas e a quantidade de vezes que foram utilizadas tanto corretas, quanto incorretamente na sua solução.

Um OAH pode operar em modo stand-alone, sem estar conectado aos servidores da plataforma. Neste caso o OAH mantém os históricos das atividades em um arquivo temporário, que é transferido para o agente Monitor no momento da próxima conexão à plataforma, de forma a manter atualizado o log de interações do SAAPIEnS.

O agente Analyzer é o responsável pelo pré-processamento dos dados e geração 
das análises estatísticas. Sua principal função é gerar os resultados que servirão de subsídios ao professor, para que o mesmo possa fazer o acompanhamento do desempenho dos alunos, assim como desenvolver ações pedagógicas visando contribuir na melhoria do processo de ensino.

Os agentes do SAAPIEnS interagem com agentes do Heráclito através de troca de mensagens e de recursos entre os mesmos. Na Figura 5, é possível visualizar o ambiente da plataforma e de interação entre os agentes do sistema. O agente Mediador é responsável pelo acompanhamento de todo o processo de tutoria, de acordo com a parametrização definida na autoria de $\mathrm{OAH}$, o professor poderá habilitar o grau de granularidade de apoio do serviço de tutoria [Vanlenhn,2006]. O agente Especialista em dedução natural é o responsável pela análise das provas realizadas pelos alunos podendo gerar automaticamente provas de dedução completas, mas também é capaz de finalizar provas parciais, ou seja, completar as provas que estão sendo desenvolvidas pelo aluno. O agente Modelo do aluno é responsável por receber as ações e manter informações relacionadas ao aluno, assim como fazer a troca de informações com o agente Monitor.

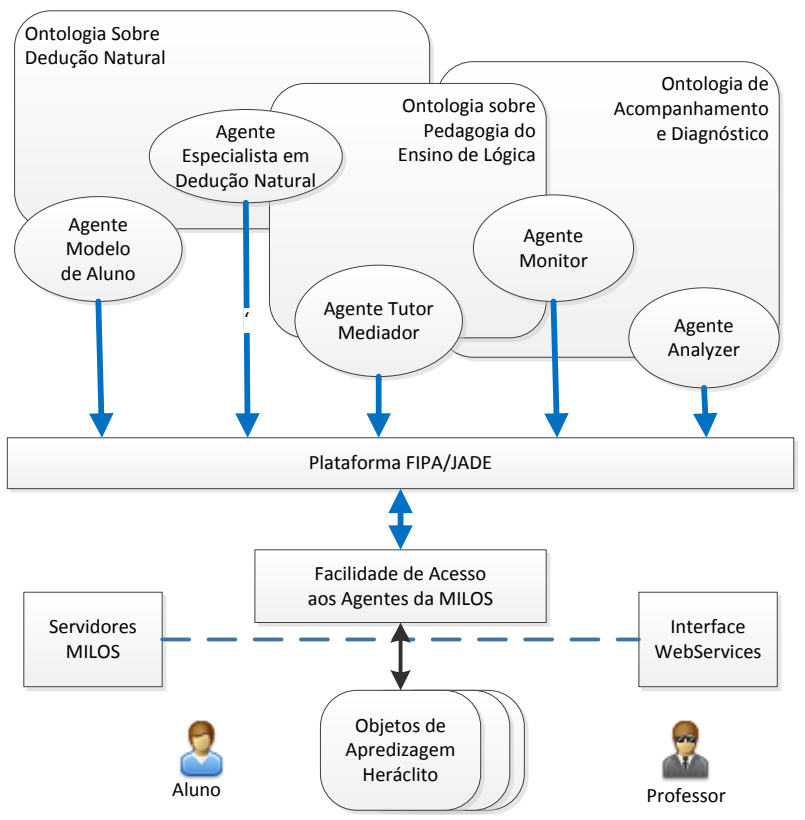

Figura 5. Integração da Plataforma SAAPIEnS-Heráclito

No caso dos $\mathrm{OAH}$, o acesso aos agentes do sistema de apoio pedagógico é disponibilizado por meio de uma interface WebService. Os OAH implementam um cliente de serviços, que acessa o provedor de serviços Web que implementa mecanismos de comunicação que convertem as requisições de serviços dos $\mathrm{OAH}$ que podem então ser enviadas aos agentes pedagógicos do Heráclito por meio da plataforma de comunicação JADE [Bellifemine, 2010].

\section{Cenário de Experimentação}

O cenário de teste aqui relatado, compreende quatro fases distintas sendo elas:1Criação e Disponibilização do OAH 2-Resolução dos Alunos; 3-Monitoramento, avaliação e análises 4- Ações previstas .

Na geração do OAH, é definido um conjunto de 10 questões de DNLP (Dedução Natural na Lógica proposicional), previamente classificadas e catalogadas no banco de 
questões pelo professor, abrangendo 4 (quatro) níveis de dificuldades. 2 (duas) do nível Fundamental, 3 (três) Básicas, 3 (três) Intermediárias e 2 (duas) Avançadas, além de dispor de um material de atividades extras, contendo um texto sobre regras de inferências de DNLP e exemplos de resolução utilizando as regras derivadas. Cabe ressaltar, que esses níveis foram definidos inicialmente, obedecendo alguns critérios básicos sendo eles: (a) $\mathrm{N}^{\circ}$ de Hipóteses; (b) $\mathrm{N}^{\mathrm{o}}$ de passos na sua resolução; (c) $\mathrm{N}^{\circ}$ de regras utilizadas e por último, (d) Tempo médio de resolução. Esses critérios tiveram como base, estudos realizados com professores da área, comparando as suas resoluções, com a do Tutor ficando então, assim categorizadas. Uma vez criado e validado pelo professor, o OAH é então disponibilizado a um conjunto de 5 (cinco) alunos que já possuíam conhecimento do conteúdo, com um prazo determinado para sua conclusão.

O acompanhamento se deu através do monitoramento dos logs de acessos, após o processo de consolidação dos dados, no momento em que o professor realizou a consulta na base do SAAPIEnS. É possível então, perceber através de análise comparativa, que os exercícios considerados de baixo e médio grau de dificuldade foram resolvidos dentro dos parâmetros iniciais, com uma pequena variação. Porém os exercícios de nível intermediário e avançado apresentaram diferenças com as questões de maior dificuldade ficando dois alunos sem resolver uma questão e outro sem resolver duas questões.

Com isso foi possível verificar o tempo dispendido pelos alunos em cada questão, o tempo que eles permaneceram conectados à plataforma do SAAPIEnS, o $\mathrm{n}^{\circ}$ de tentativas em cada solução, $\mathrm{o}^{\mathbf{0}}$ de solicitações de ajuda e quais as regras de dedução que apresentaram maiores dificuldades. Todas essas informações serviram de subsídios ao professor, para detectar o grau de dificuldade encontrado pelos alunos. Sendo assim foi possível definir um conjunto de ações pedagógicas que visaram atender, especificamente aos alunos que apresentaram maior grau de dificuldade na resolução dos exercícios. Estas ações foram: (a) Propor um novo OAH com questões que envolvessem o conjunto das regras de DNLP que os alunos não conseguiram resolver, porém com diversos graus de dificuldade, além de disponibilizar atividades extras como apoio aos alunos. (b) Submeter novamente o OAH e avaliar novamente o desempenho dos alunos na sua resolução.

\section{Considerações Finais}

O uso de tecnologias nos contextos educacionais tem contribuído de maneira substancial no processo de ensino-aprendizagem nos últimos anos. $\mathrm{O}$ crescimento da web como fonte de informações e a quantidade de recursos disponíveis tanto a alunos quanto para professores, fazem desse cenário um ambiente promissor para pesquisa e $\mathrm{o}$ desenvolvimento de novas ideias no âmbito da educação. Nesse contexto é preciso levar em consideração a importância que o professor representa dentro do cenário educacional, tanto como especialista em ensino, quanto como um usuário de ambientes digitais, sendo, portanto, imprescindível provê-lo de estruturas que contribuam para a melhoria do seu trabalho.

Espera-se que o conjunto de recursos de criação, acompanhamento e análise do uso de OA fornecido pelo SAAPIEnS permita aos professores avaliar o desempenho alcançado pelos alunos de uma maneira significativa, permitindo que se possa, não só realizar ações corretivas, como também prever situações futuras e agir pro-ativamente. O SAAPIEnS não foi concebido com objetivo de um ciclo único de aprendizagem. À 
medida que informações de acompanhamento são atualizadas e adicionadas à base, novas análises se tornam possíveis e novas conclusões podem ser percebidas. Espera-se que isso ajude o professor a estabelecer critérios no desenvolvimento de planos de ensinos, adequados à realidade do contexto dos seus alunos. As facilidades oferecidas pelo uso desta ferramenta facilitam também o desenvolvimento dos objetos de aprendizagem inteligentes customizados especificamente para um determinado fim, visando atingir determinado grupo de alunos, podendo assim contribuir de maneira qualitativa no progresso realizado pelos mesmos.

\section{Agradecimentos}

Os autores agradecem ao MCT/FINEP/MC/FUNTTEL, a CAPES e ao CNPq pelo financiamento dessa pesquisa.

\section{Referências}

Bellifemine, F. L.; Caire, G.; Greenwood, D. (2007) Developing Multi-Agent Systems with JADE. John Wiley \& Sons.

Bez, M.; Vicari, R. M.; Silva, J. M.; Ribeiro, A.; Gluz, J. C.; Passerino, L. M.; Santos, E.; Primo, T.; Rossi, L.; Behar, P.; Filho, R.; Roesler, V. (2010) Proposta Brasileira de Metadados para Objetos de Aprendizagem Baseados em Agentes (OBAA). RENOTE, v.8, p.1 - 10.

Bordini, R., Hübner, J., Wooldridge, Michael. (2007) Programming Multi-Agent Systems in AgentSpeak using Jason. Wiley Series in Agent Technology.

Mossman, M.; Gomes, L.; Gluz, J. C. (2012) Objetos de Aprendizagem Móveis para Ensino de Dedução Natural na Lógica Proposicional. In: Anais do Simpósio Brasileiro de Informática na Educação.

Nassar, Silvia M., Wronscki, Vilson R., Ohira, Masanao et al. SEstatNet - Sistema Especialista para o Ensino de Estatística na Web. URL de acesso: http://www.sestat.net . Florianópolis - SC, Brasil. Acessado em 03/ 07/2014.

Penteado, F.; Gluz, J. C. (2011) Sistema Heráclito: Suporte a Objetos de Aprendizagem Interativos e Dialéticos Voltados ao Ensino de Dedução Natural na Lógica Proposicional. In: Anais do Simpósio Brasileiro de Informática na Educação.

Penteado, F. (2013) Agente pedagógico para mediação do processo de ensino aprendizagem da dedução natural na lógica. 2013. 127 f Dissertação (mestrado). Universidade do Vale do Rio dos Sinos, Pós-Graduação em Computação Aplicada, RS.

Vanlehn, K. (2006) The behavior of tutoring systems. International journal of artificial intelligence in education, v. 16, n. 3, p. 227-265.

Viccari, R.; Gluz, J; Passerino, L.; et al. (2010) The OBAA Proposal for Learning Objects Supported by Agents. Procs. Of MASEIE Workshop - AAMAS 2010, Toronto, Canada.

Wooldridge. M. (2009) An Introduction to MultiAgent Systems. 2nd ed. John Wiley \& Sons.

W3C World Wide Web Consortium : Authoring Tools, Social Media Disponível em:

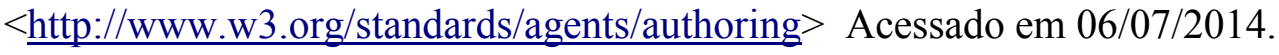

